\title{
Smartphone Controlled Multipurpose Robot Car
}

\author{
Balendu Teterbay \\ Student \\ Dept. of Electronics and Communication Engineering \\ RV College of Engineering \\ Bangalore, India \\ Ayush Srivastava \\ Student \\ Dept. of Electronics and Communication Engineering \\ RV College of Engineering \\ Bangalore, India
}

\author{
Akshay Bhati \\ Student \\ Dept. of Electronics and Communication Engineering \\ RV College of Engineering \\ Bangalore, India \\ Dr. Abhay A. Deshpande \\ Associate Professor \\ Dept. of Electronics and Communication Engineering \\ RV College of Engineering \\ Bangalore, India
}

\begin{abstract}
The aim of this project is to develop a prototype of a smartphone-controlled robot car that performs a various function in order to provide a very powerful and versatile robot while also reducing the hardware usage as much as possible. In this project, Arduino UNO is used as a central component, to which all the other components are interfaced. The designed vehicle is controlled wirelessly through a smartphone with the help of a Wi-Fi module. On detecting an obstacle, a notification is sent to the smartphone and the robot performs pick and place operation with the help of a robotic arm mounted onto the vehicle. A combination of Temperature sensor, Gas sensor and Fire sensors are used to provide explosion sensing and detection and a buzzer goes on with the detection of explosion prone region. Further, a live surveillance is provided to monitor every movement of the vehicle.
\end{abstract}

Keywords_IOT; Arduino UNO; Node MCU; WiFi

\section{INTRODUCTION}

With the increase of technological advancements, everyone wants multitask-enabled products. In the past, numerous robotic cars were built to perform certain type of tasks. With this project, a wide scope of capacities is incorporated into a solitary model of a military automated vehicle. It is the robot which autonomously detects obstacles in front of it and does pick and place function[5], along with explosion sensing and video surveillance. It uses the flame sensor, gas sensor, temperature sensor for detection purposes. The robot can move freely based on the controls given to it via a smartphone while actively scanning for the fire, gas and temperature. For this purpose, variety of sensors are mounted onto the vehicle. When an obstacle is detected, the robot can indicate into IOT Site. The aim of this project is to develop a prototype of a smartphone-controlled robot car that performs a various function in order to provide a very powerful and versatile robot while also reducing the hardware usage as much as possible. In this project, Arduino UNO is used as a central component, to which all the other components are interfaced. The designed vehicle is controlled wirelessly through a smartphone with the help of a Wi-Fi module[2]. On detecting an obstacle, a notification is sent to the smartphone and the robot performs pick and place operation with the help of a robotic arm mounted onto the vehicle. A combination of Temperature sensor, Gas sensor and Fire sensors are used to provide explosion detection[6] and a buzzer goes on with the detection of explosion prone region. Further, a live surveillance is provided to monitor every movement of the vehicle. Through detecting explosion-prone areas and signaling the same to the smartphone, the robot will save much life. It can be useful in detecting other forms of accidents where the atmosphere is very dangerous to humans, such as operating around toxic or radioactive products or the easily explodable propane tank. The robot can be used for surveillance because they can easily cover areas where humans are unable to reach, and then automatically execute their functions when there are obstacles.

\section{LITERATURE REVIEW}

In this paper, a robot that extinguishes fire in real time is designed to extinguish the fire in the event of a fire disaster. It consists of two fire sensors that sense fire and then relay the signal to the module for motion control. The robot is then designated using a modern GSM to move to the fire location and extinguish the fire and also alarm the customer via a cell phone. To ease the movement of the robot, sets of IR sensors are used to facilitate the movement in free space and to avoid obstacles. The robot is able to communicate with GSM modern by using an RS232 interface and with remote supervise computer using Internet [1].

In this paper, a robot is designed to allow it to follow a black line path while accommodating various other features such as collision detection and avoidance or falling from a certain height with great stability and control. The design is incorporated with IR sensors, Bluetooth and Wi-Fi modules interfaced with a central Microcontroller Arduino UNO. The robot is controlled by used end connected through long range $\mathrm{Wi}-\mathrm{Fi}$ connectivity and the path of the car can be changed from the used side [2].

A Bluetooth empowered mechanical vehicle is planned and afterward it gives a point by point similar examination of different sensors utilized in apply autonomy. The planned vehicle is controllable by utilizing a Bluetooth module and a mechanical knock sensor is connected on the facade of the vehicle to decide whether an impact has happened to it likewise gives the specific time of crash [3]. 
A mechanical vehicle is structured with the assistance of a Microcontroller Arduino UNO. The vehicle is wirelessly controlled with the help of a Bluetooth module. The activity of the structured vehicle is separated into two portions. One deals with wireless communication using an application, and the other deals with avoiding collisions. On detecting any object within a threshold distance, the car stops and analyze all directions forward, backward, right and left. Whichever provides more clearance, the car moves in that direction [4].

In this paper, a pick and place robotic car is designed with a purpose of picking the obstacle in front of it and placing aside. Ultrasonic sensors are used for detection purposes. A Java programmed application is developed to give instructions to the microcontroller. A camera is placed on the car for surveillance, which clicks the picture of the obstacle which is then sent to the microcontroller so that the car is moved to perform pick and place function. Further EM induction concepts are used to provide wireless charging facility when the battery goes low [5].

A mine detection robot is built in this paper with the help of different sensors. For detection purposes, a combination of ultrasonic sensor, gas sensor, temperature sensor, humidity sensor is used. The system is designated to assist the military in places where mine detection is highly probable. Further, the designed car is accompanied with a camera for video surveillance and an alert is sent to a smartphone with the help of IOT whenever a mine is detected. Thus, the robot proves to save the lives of military personnel on the front lines by replacing them wherever possible [6].

\section{PROPOSED SYSTEM}

A smartphone-controlled robot car is structured using Arduino UNO. With the aid of a variety of sensors listed below, the designed car performs diverse functions. An ultrasonic sensor with the assistance of a robotic arm which is placed on the robot car is used for picking and placing any obstacle in front of the car. For the explosion sensing, a combination of Gas sensor, Fire Sensor and Temperature sensors are used. Further a Wi-Fi module is used to establish wireless connectivity for controlling the robot car through a smartphone. The major part of the project is the design and development of automated gripper control for robotic arm. Gripper will be created and attached to the robot as a method to move the obstacles from one place to another, according to

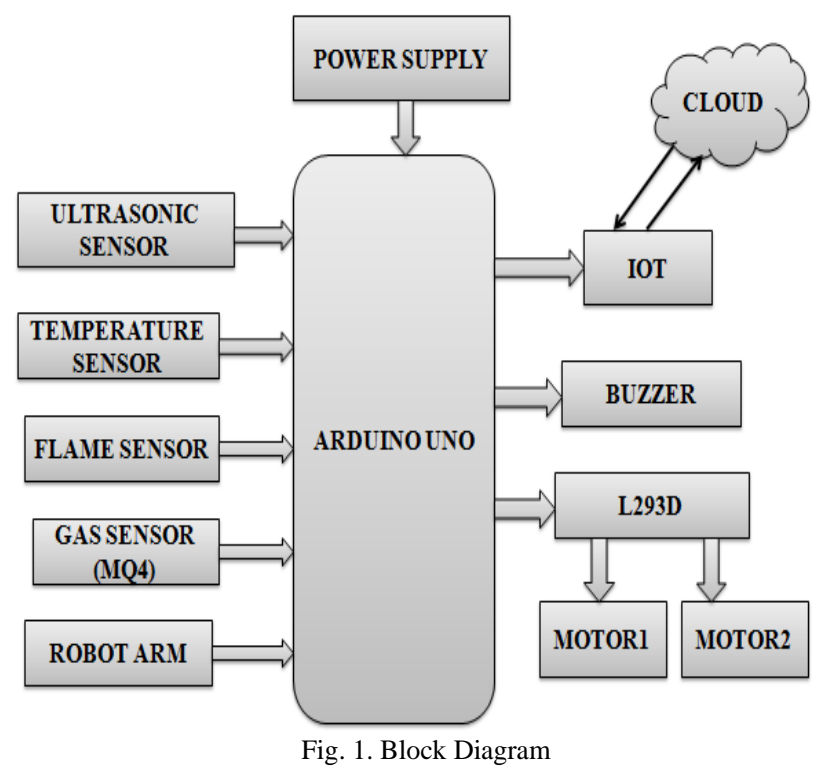

the input program and the place to move it. The robot vehicle is created with significant spotlight on four key segments which is the mechanical part, the gripper, electrical part and a controller. Each part is planned independently stage by stage. Here Temperature, Gas Sensor and Flame sensor is interfaced with the controller. The measured sensor details are monitored through IOT. The robot works based on the command obtained through IOT. There is a buzzer attached which goes on with the detection of any obstacle and an alert will be sent to the smartphone to acknowledge that an obstacle has been detected. Alongside this there will be a camera appended to the vehicle to convey live video surveillance to screen the movement of the vehicle. A simulation is performed to check the authenticity of the Arduino codes. For this, Proteus Isis Professional Simulation software is used. Below is the block diagram in Fig. 1 mentioning the components and functions of the designed vehicle.

\section{Arduino UNO}

The Arduino UNO is an open source hardware development board and is based on the Microchip ATmega328P microcontroller. The board is furnished with a mix of input-output pins, both analog and digital. The pins are utilized to interface the Arduino board with different shields and circuit boards. The board comprises of 14 digital pins and 6 analog pins and it is programmable with an integrated development system called Arduino IDE with the assistance of a B type USB link. It very well may be fueled by 9 volt outer battery, in spite of the fact that it acknowledges voltages in the scope of 7 to 20 volts.

\section{Ultrasonic Sensor}

An electronic device that measures the distance of a target object by emitting ultrasonic sound waves and eventually turning the sound, which is reflected back into an electrical 


\begin{tabular}{|c|c|c|c|c|c|}
\hline Component & $\begin{array}{c}\text { Power } \\
\text { Supply }\end{array}$ & $\begin{array}{c}\text { Operating } \\
\text { Voltage }\end{array}$ & $\begin{array}{c}\text { Operation } \\
\text { Temperature }\end{array}$ & Measurements & Clock Speed \\
\hline Arduino UNO & $3.3 \mathrm{~V} / 5 \mathrm{~V}$ & $5 \mathrm{~V}$ & -40 to $85^{\circ} \mathrm{C}$ & -- & $16 \mathrm{MHz}$ \\
\hline Ultrasonic sensor & $+5 \mathrm{~V} \mathrm{DC}$ & 4.5 to $5.5 \mathrm{~V}$ & -25 to $70^{\circ} \mathrm{C}$ & $\begin{array}{c}2 \mathrm{~cm} \text { to } 400 \mathrm{~cm} \\
\text { distances } \\
-55^{\circ} \mathrm{C} \text { to } 150^{\circ} \mathrm{C} \\
\text { temperature range } \\
760 \text { to } 1100 \mathrm{~nm} \\
\text { wavelengths }\end{array}$ & $16 \mathrm{MHz}$ \\
\hline Temperature sensor & $+5 \mathrm{~V}$ & $5 \mathrm{~V}$ & -55 to $150^{\circ} \mathrm{C}$ & $\mathrm{MHz}$ \\
\hline Fire sensor & $5 \mathrm{~V}$ & 3.3 to $5 \mathrm{~V}$ & Less than $73.8^{\circ} \mathrm{C}$ & $\begin{array}{c}200 \text { to } 10000 \mathrm{ppm} \\
\text { concentration }\end{array}$ & $20 \mathrm{MHz}$ \\
\hline Gas sensor & $+5 \mathrm{~V}$ & $+5 \mathrm{~V}$ & -10 to $-50^{\circ} \mathrm{C}$ & -- & $80 \mathrm{MHz}$ \\
\hline Node MCU & $3.3 \mathrm{~V}$ & 2.5 to $3.6 \mathrm{~V}$ & -40 to $125^{\circ} \mathrm{C}$ & & \\
\hline
\end{tabular}

Table.1. Specifications

signal is known as an ultrasonic sensor. Active ultrasonic sensors generate high-frequency sound waves and evaluate the echo, eventually being received back by the implemented sensor, which then measures the time interval between sending of the signal and receiving of the echo to determine the distance to an object. For a fact, Ultrasonic waves travel faster than the speed of the normal sound which is audible. Transmitter and Receiver are the two main components of an ultrasonic wave sensor, These devices work on a principle which is similar to that of transducers used in radar and sonar systems, which helps in evaluating attributes of a target by interpreting the echoes from radio or sound waves, respectively.

\section{Fire Sensor}

A device made and designed to respond after detecting to the presence of a flame or fire is known as a fire sensor. When fire burns it emits a small amount of Infra-red light, which is eventually received by the Photodiode (IR receiver) on the sensor module. Then an Op-Amp is used to check for change in voltage across the IR Receiver, such that in case a fire is detected, the output pin, D0, will give LOW and if the is no fire the output pin will be HIGH.

\section{Temperature Sensor}

A device which is made with a motive to measure the hotness or coldness of an object is known as a temperature sensor. An example of a precision IC temperature sensor which has its output being rationally proportional to the temperature (in ${ }^{\circ} \mathrm{C}$ ) is LM35. The temperature can be measured more precisely than with a thermistor using LM35. It has low self-heating and it does not push the temperature rise higher than $0.1^{\circ} \mathrm{C}$ in air. The range foe operation is $-55^{\circ} \mathrm{C}$ to $-150^{\circ} \mathrm{C}$.

\section{Gas Sensor}

A gas sensor is a type of instrument which is used to identify the presence of various types of gases available in the sensing range of a gas sensor. This sensor detects the presence of gas and performs the action programmed for it. A gas sensor can notify the user when the leak is occurring, giving them the opportunity to leave. This type of device is essential because unknown gases can affect living and nonliving things. Gas sensors can be programmed to notify oxygen concentration present in the air and flammable and dangerous gases. This type of device has a vast area of exploit and is used in various place such as industries, houses etc.

\section{Node $M C U$}

Node MCU is a firmware which works on a open source platform that helps to prototype or build IoT products. MCU stand for microcontroller unit which means that it is like a computer running on just a single chip. Its firmware is based on ESP8266 Wi-Fi SoC from Espressif Systems and its hardware is based on the ESP-12 module. A SoC i.e System on a Chip is a computer or electronic instrument comprising of all its component miniaturized on a single chip. ESP8266 is a very low-cost chip available and has added functionality which supports Wi-Fi module chip that on user's command can connect to the internet for Internet of Things (IoT) and it has other functionalities as well. The firmware used Lua scripting language and is based on eLua project and is built on the Espressif Non-OS SDK for ESP8266. LUA programming language or Arduino IDE are often used for programming purposes of the chip via a USB port. Just like an Arduino, it can easily be programmed to our needs such as initiating a Wi-Fi connection and defining various things such as $\mathrm{I} / \mathrm{O}$ pins etc. It can used in place of a Ethernet module according to the convenience. It merges the characteristics of station microcontroller and a $\mathrm{Wi}-\mathrm{Fi}$ access point. The above attributes make it an extremely efficient Wi-Fi networking tool. It can be programmed accordingly to act such that it performs the functionality of a access point or even a station, host a web server or connect to internet to fetch or upload data.

\section{RESULTS}

The designed smartphone-controlled pick and place model is shown in Fig.2. All the sensors are interfaced with Arduino board and fitted in the chassis using jumper wires. The gripper is attached at the top as shown. The simulation of the project is performed in Proteus Isis Professional Simulation software. The image of the simulation in the software along with the virtual window is shown in Fig.3. The vehicle is controlled with instructions given by the user. Fig.3 shows the controls used to give commands to the vehicle in the Cayenne software. Cayenne is an online IOT dashboard. The 
screen shows forward, backward, right, and left options to control the vehicle. Also, fire, temperature and gas symbols are there which indicates, if any of these are detected or not by the sensors in the vehicle.

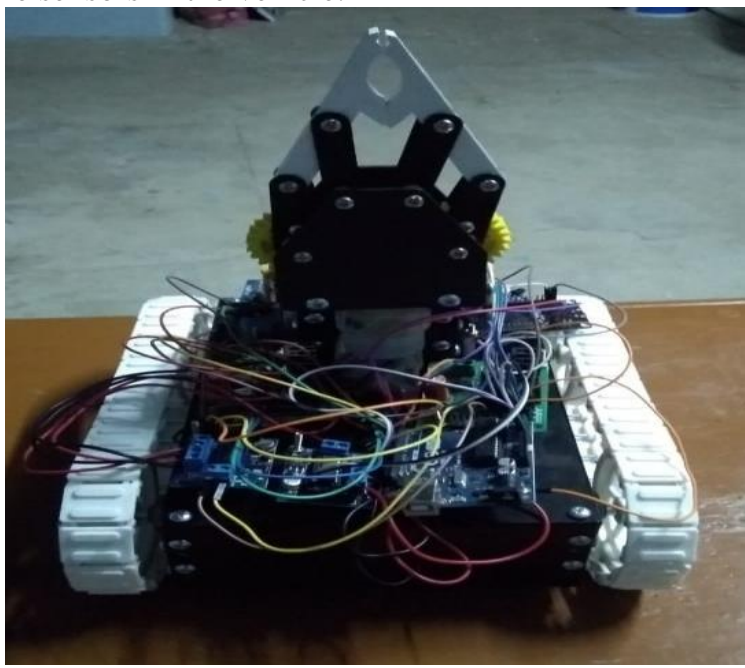

Fig. 2. Smartphone Controlled Hardware Model

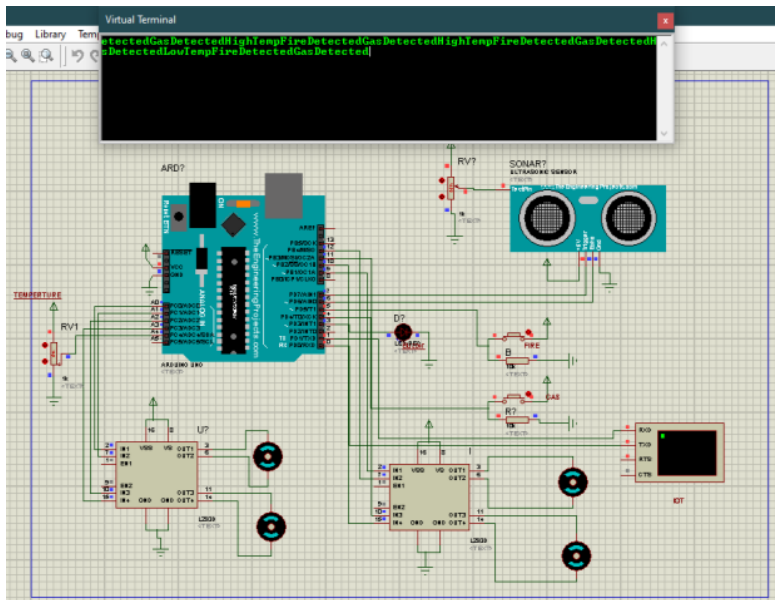

Fig. 3. Simulation in Proteus Simulation software

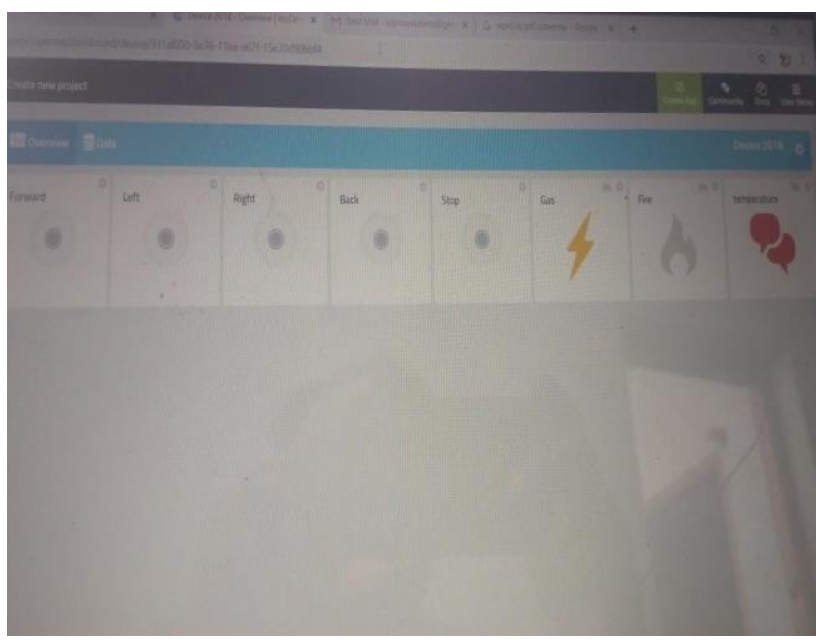

Fig. 4. Controls in Cayenne Software

\section{FUTURE SCOPE}

This model structure is merely the foundation of a fundamental execution. This sector provides an enormous amount of increase for potential work and study. The general structure has room for improvement, with the goal of being able to function and adapt in various requirements of circumstances. The module can be additionally reached out to acknowledge voice as a contribution to give guidelines to the designed vehicle.

\section{CONCLUSION}

In this project, the field of Robotics is investigated and a robotic car equipped with many functionalities is proposed. The designed model detects any obstacle encountered in front of it and places it aside. Along with this, explosion sensing is achieved by using a combination of sensors such as Gas sensor, Fire sensor and Temperature sensors.

During the project, the complete working of Arduino UNO and a variety of sensors is executed and understood. Coding and designing skills form the base and are utilized in the project. The project gives profound knowledge into different advancements and devices for improvement of the venture. Exposure to various software such as Arduino IDE and Proteus widened the knowledge in a broad aspect. There is much scope of improvement in the communication range and considerable reduction in processing time.

The use of Wi-Fi technology instead of the more widely used Bluetooth increases the range of communication and improves the overall performance of the designed model.

\section{REFERENCES}

[1]. T. L. Chien, H. Guo, K. L. Su and S. V. Shiau, "Develop a Multiple Interface Based Fire Fighting Robot," 2007 IEEE International Conference on Mechatronics, Changchun, Jilin, 2007, pp. 1-6, doi: 10.1109/ICMECH.2007.4280040.

[2]. S. Mandal, S. K. Saw, S. Maji, V. Das, S. K. Ramakuri and S. Kumar, "Low cost arduino wifi bluetooth integrated path following robotic vehicle with wireless GUI remote control," 2016 International Conference on Information Communication and Embedded Systems (ICICES), Chennai, 2016, pp. 1-5.

[3]. H. Rissanen, J. Mahonen, K. Haataja, M. Johansson, J. Mielikainen and P. Toivanen, "Designing and implementing an intelligent Bluetoothenabled robot car," 2009 IFIP International Conference on Wireless and Optical Communications Networks, Cairo, 2009, pp. 1-6.

[4]. Zhao Wang, Eng Gee Lim, Weiwei Wang, M. Leach and Ka Lok Man, "Design of an arduino-based smart car," International SoC Design Conference (ISOCC), Jeju, pp. 175-176, 2014

[5]. N. Firthous Begum, P. Vignesh. Design and Implementation of Pick and Place Robot with Wireless Charging Application, International Journal of Science and Research (IJSR), ISSN (Online): 2319-7064, 2013.

[6]. Zhenjun He, Jiang Zhang, Peng Xu, Jiaheng Qin and Yunkai Zhu, "Mine detecting robot based on wireless communication with multisensor," 2013 IEEE 4th International Conference on Electronics Information and Emergency Communication, Beijing, 2013, pp. 117120

[7]. Y. Zhang, B. K. Chen, X. Liu and Y. Sun, "Autonomous Robotic Pickand-Place of Microobjects," IEEE Transactions on Robotics, vol. 26, no. 1 , pp. 200-207, 2010

[8]. S. S. Pujari, M. S. Patil and S. S. Ingleshwar, "Remotely controlled autonomous robot using Android application," International Conference on I-SMAC (IoT in Social, Mobile, Analytics and Cloud) (I-SMAC), Palladam, pp. 588-593, 2017

[9]. D. Singh, P. Zaware and A. Nandgaonkar, "Wi-Fi surveillance bot with real time audio \& video streaming through Android mobile," 2017 2nd IEEE International Conference on Recent Trends in Electronics, Information \& Communication Technology, Bangalore, pp. 746-750, 2017

[10]. T. Kaur and D. Kumar, "Wireless multifunctional robot for military applications," 2015 2nd International Conference on Recent Advance in Engineering \& Computational Sciences (RAECS), Chandigarh, 2015, pp. 1-5, doi: 10.1109/RAECS.2015.7453343. 